\title{
Myorelaxation in the training process of skilled athletes
}

\author{
Yuriy P. Denisenko ${ }^{1}$, Yuriy V. Vysochin ${ }^{2}$, Leonid G. Yatsenko ${ }^{2}$ \\ ${ }^{1}$ Department of Theory and Methods of Sports Games, Branch of Povolzhskaya State, Academy of Physical Culture, Sports and \\ Tourism, Naberezhnye Chelny, Russia \\ ${ }^{2}$ Department of Physical Education, St. Petersburg State Technological University of Vegetative Polymers, St. Petersburg, Russia \\ Email: yprof@yandex.ru
}

Received 14 January 2013; revised 16 February 2013; accepted 24 February 2013

Copyright (C) 2013 Yuriy P. Denisenko et al. This is an open access article distributed under the Creative Commons Attribution License, which permits unrestricted use, distribution, and reproduction in any medium, provided the original work is properly cited.

\begin{abstract}
At the present time, a number of various ways of sportspersons' special physical capability (SPC) based mainly on training and competitive loadings ramp up. They are effective enough to reach the main goal, but none of them provides sportspersons' health safety. Moreover, with the increase in volume and intensity of the loadings, which in sport have almost reached their limits, the sport traumatism and morbidity rate grow progressively. Proceeding from this, there was an evident necessity for the search of conceptually new ways for a simultaneous solution of these two the most complex and, in the opinion of many research workers, almost incompatible problems - the problem of achieving the highest levels of special physical working capacity, and the problem of sportspersons' health maintenance and improving-associated by us into one general problem of human motor activity efficiency enhancement. Therefore it is necessary to physiologically substantiated the basic methods and principles of special relaxation training, directed on increase of efficiency of process of training of sportsmen at all stages of the development of athletic skills. Under the efficiency of the motor activity, we understand achieve the highest levels of the special physical performance while maintaining sports longevity and health of athletes.
\end{abstract}

Keywords: Extreme Conditions; Protection Functional System; Muscle Relaxation Rate; Physical Efficiency; Central Nervous System; Relaxation

\section{INTRODUCTION}

Professional tendencies of the last years are connected with steady growth of loadings in practically all kinds of human professional activities. The consequence of this is often the disturbance in the work of regulatory mechanisms, that essentially decreases the level of physical capability and can result in various unfavorable vegetative shifts in health state [1-3], the problem of providing effective training of sportspersons in extreme conditions of life activity and creating functional preconditions for health saving being more and more topical. One of the ways to solve this problem is attracting modern effective and physiologically substantiated technologies with the simultaneous use of the functional state correction and complex diagnostics rational system. Such an approach allows widening the diapason of compensatory abilities of the body against the maximal volume and intensity of professional and psycho-emotional loadings. The provision of optimal adaptation to muscular loadings can appear one of the conditions for the health level maintenance and professional mastery quality increase [2,4-6].

Certainly, the given problem acquires a special meaning in modern conditions of the human professional activity. It finds its reflection in a series of works connected with the idea of loading criticism both in sport and other areas of professional activity [6,7].

Together with traditional approaches a great experience of using a whole range of non-traditional means (baro-chamber, hypoxic and hyper-pyretic effects, special breathing exercises, methods of biological feedback, methods of active self-adjustment and relaxation, etc.) within the system of sport training has been accumulated. Together with that it is necessary to note that among the non-traditional means of effect on the functional state of the human body a careful attention has lately been paid to myorelaxation methods, which such features as action safety, relative easiness of effect achieving and not high financial expenditures are typical of. Relaxation, on some authors' opinion, is considered as an alternative or compliment to the functional state correction [8-10]. That is why it is often presented as a means of prophylaxis, correction and emotional stresses elimination. Thereat, as 
many note [10], it is the leading one in the series of methods allowing achieving necessary changes in the body's functional state.

In physiology an active process of muscular tone and psycho-emotional tension decrease $[9,11,12]$ are meant by relaxation. At relaxation there appears a trophotropic state, the level of anxiety, psychological and physiological response to stress effects decreases. Besides, relaxation is attended by a considerable reduction of afferent and efferent impulsation. As a consequence we can speak on the fact that the introduction of relaxation methods aimed at the prophylaxis, correction and negative psycho-emotional states elimination into practice can promote adaptive capabilities of the body [2,13,14].

The relaxation methods have also found their application in the correction of a range of pathological states, hypertensive disease treatment, acute and chronic painful states taking down inclusive of sport activity $[10,15,16]$.

The state of relaxation lies in the foundation of meditative methods. Meditation and relaxation exercises have a wide diapason of application, most often they are used in transcendental medicine $[4,9,11,13]$.

The value of muscles relaxation function in human sport and labour activities is difficult to overestimate. In a series of works $[2,8,13,17-19]$ a healthy influence of special exercises enhancing the function of skeletal muscles relaxation on the central nervous system, visceral organs' and systems' activities, rational blood circulation types formation, motion coordination, tempo, stamina, technical skills, special physical working capacity and sport results growth were proved.

The investigations proving the leading role of inhibitory systems of the central nervous system and skeletal muscles' arbitrary relaxation rate (ARR) in the most important manifestations of life activity of the whole body: in the mechanisms of timed and long-tern adaptation to more physical, hypoxic and hyper-pyretic loadings; in the mechanisms of heart adaptation and various blood circulation types formation; in the mechanisms of muscles blood supply and muscular activity energy supply; in the mechanisms of physical overwork stability improving, prevention of risks, traumas and diseases, and also in the body's mechanisms of defence from extreme conditions or factors and sportspersons rehabilitation $[7,10,16]$, are especially meaningful, in our opinion.

It should also be noted that all the most effective methods of psycho-regulation, self-adjustment and autotraining used in special psychological preparation of sportspersons and the latest health-improving technologies $[9,11,14]$ are based on relaxation.

\section{METHODS}

To study the mechanisms of regulation and coordination of arbitrary movements control the contractile and some relaxation characteristics of skeletal muscle, functional state of the central nervous system (CNS) and neuromuscular (NMS) systems we used the method of computer polymiografy, designed by Y. Vysochin, which is used for preparation of sportsmen of national teams of Russia and St.-Petersburg. Marked indicates its high informativeness and reliability.

Polymyography is based on synchronous graphic recording of the bioelectric activities and strengths (in the form of the èlektomyogram and the dynamogram, respectively) of the quadriceps muscles of both thighs during their maximally rapid and strong contraction and relaxation in the isometric mode. When deciphering polymyograms, we estimated the rates of the motor reactions of contraction and relaxation; the rate of development and the strength of excitatory and inhibitory processes in the CNS; the inhibition-excitation balance in the CNS; the relative rate of voluntary muscle contraction, or the so-called explosive characteristics of muscles; the relative maximum voluntary muscle strength; the rate of voluntary muscle relaxation; and the general functional states of the muscles, CNS and NMS. In addition, we used the ratio between the relative maximum voluntary muscle strength and the rate of voluntary muscle relaxation to calculate the classification index of the type of long-term adaptation or individual development.

\section{RESULTS AND DISCUSSION}

Summarizing the results of our longstanding research we can substantiate the main ways and principles of special relaxation training, aimed on higher effectiveness of training process of sportsmen at all stages of development of sport skills. Under effectiveness of motional activity we understand achievement of the highest levels of special exercise performance (SEP) at condition of total preservation and improvement of sportsmen's health.

Nowadays there arc known different means of sportsmen's SEP improvement, based basically on increasing of training and emulative loads. They are quite effective for achieving the main target, but none of them provides preservation of sportsmen's health. Moreover, when the amount and intensity of exercise stress increase, sport traumatism and morbidity increase progressively. As well as the amount and intensity of exercise stress in sport almost reached their limits already. There are well known different means of health improvement; most of them consider moderate physical activity of low intensity to have the leading health-improving role. However this approach does not contribute to progress in special exercise performance and sport results. That is why it was obviously necessary to find fundamentally new ways to solve these two very complicated problems at the same 
time- - how to achieve the highest levels of special exercise performance and how to preserve and improve sportsmen's health. In opinion of many investigators, these problems arc almost incompatible. We have combined these problems into one common problem-to make man's motional activity more effective. In several sets of experiments, in which sportsmen of different levels of proficiency and different specialization took part, we have found direct significant dependence between SEP and, of course, sport results and velocity of voluntary relaxation (VVR) of skeletal muscles [11]. In most kinds of sport (in 17 from 20) meaning of SEP in the progress of sport results, especially at the stage of higher sport mastery was considerably higher then meaning of contractile properties of muscles. In such kinds of sport as box, hockey, football, skating, decathlon and swimming SEP was not only leading, but also the only one of all polimiographic parameters, which defines qualification growth. In some kinds of sport (football, skating, decathlon, swimming), for example, maxrnal muscles force of the sportsmen of high level of proficiency was slightly (not authentic) lower, than at the sportsmen of lower level of proficiency. This fact in no way means that contractile properties of muscles do not play any role in efficiency mechanisms. On the contrary, they are very important because muscles contraction provide physical action. And duration of this work, i.e. exercise tolerance and, correspondingly, special exercise performance considerably depend on relaxation characteristics of muscles. That is why our data should be considered only as a proof of the fact that the level of development of muscles contractile properties, acquired, for example, by candidates to master of sports and 1-grade sportsmen in the process of long-term sport training, is already sufficient for achieving the top of sport skills; and achievement of this top is limited mainly by the level of muscles VVR.

The above-mentioned facts, in our opinion, are quite important for understanding of the role of miorelaxation in increase of SEP in all kinds of sport activities, because in each of them there are very high requirements in velocity, velocity tolerance or coordination, or different combination of these qualities, which directly depend on muscles VVR.

However the most important role in understanding and interpretation of physiological mechanisms of SEP and tolerance to physical activity, especially in extreme conditions, is played by common nonspecific inhibitoryrelaxation functional protective system (IRFPS) of organism against extreme impacts and influence of its activity (capacity) on forming of three different types of long-term adaptation. Experimentally big advantages of relaxation type of long-term adaptation were proved; this type of adaptation develops at sportsmen with high VVR of muscles and highly active IRFPS, and it provides achievement of the highest levels of exercise performance and preservation of health in extreme conditions at the same time. We also ascertained that heightened excitability of CNS is the main factors limiting the capacity of IRFPS $[3,11]$.

\section{CONCLUSIONS}

The above-mentioned facts, in our opinion, are quite important for understanding of the role of miorelaxation in increase of SEP in all kinds of sport activities, because in each of them there are very high requirements in velocity, velocity tolerance or coordination, or different combination of these qualities, which directly depend on muscles VVR.

It should also be said here about the ARR highly authentic correlation relationships with all the principal components of motion coordination and sport results in various sports. The data for a significant influence of the ARR on the contractile muscles' properties realization degree also deserve attention. The enumerated facts, from our point of view, are meaningful enough to understand that important role, which is played by myorelaxation in the special physical capacity growth in all kinds of sport activity and sportspersons' health maintenance.

\section{REFERENCES}

[1] Balsevich, V.K. (2000) Human ontokinesiology. Theory and Practice of Physical Culture, 275.

[2] Vysochin Yu, V. (1988) Physiological mechanisms of defence, stability and physical working capacity improving in extreme conditions of sport and professional activity. Thesis of Doctor of Science, 550.

[3] Denisenko Yu, P. (2007) Relaxational characteristics of skeletal muscles in increase of physical working capacity of football players. Vestnik Sportivnoi Nayki, 1, 27-30.

[4] Vysochin Yu, V. and Lukoyanov, V.V. (1997) Active myorelaxation and self-adjustment in sport. Monograph-SPb, 85.

[5] Denisenko Yu, P. (2007) Myorelaxation in football players' training system. Synopsis of Thesis of Doctor of Science (Biology), 48.

[6] Platonov, V.N. (1988) Adaptation in sport. Zdorovye, Kiev, 257.

[7] Vysochin Yu, V. and Denisenko Yu, P. (2002) Modern ideas on physiological mechanisms of terminable adaptation of sportspersons' body to physical loadings effects. Theory and Practice of Physical Culture, 7, 2-6.

[8] Aivazyan, T.A. (1988) Relaxation therapy using biological feedback in treatment of hypertension patients. Biomanagement, Theory and Practice, Novosibirsk, 133- 141.

[9] Sentyabrev, N.N. (2004) Body's directed relaxation at intensive human muscular activity. VSAPC, Volgograd, 142. 
[10] Tkhorevsky, V.I. (1967) Blood supply of skeletal muscles at static and dynamic work. Synopsis of Thesis of Canadian Science (Medicine), 24.

[11] Vysochin Yu, V. and Denisenko Yu, P. (2010) Health as national property. Collective Monograph, 669.

[12] Huxley, H.E. (1969) The mechanism of muscle contraction. Science, 164, 1356-1366. doi:10.1126/science.164.3886.1356

[13] Vysochin Yu, V., Denisenko Yu, P. and Yatsenko, L.G. (2011) The contemporary concepts of structural and functional organization of the neuromuscular system and of the contraction and relaxation mechanisms of skeletal muscles. http://kamgifk.ru/magazin/journal.htm

[14] Sudakov, K.V. (1987) Main principles of functional systems' general theory. Functional Systems of the Body: Guidance, 26-49.
[15] Bayevsky, R.M. and Motylyanskaya, R.Ye. (1986) Cardiac rhythm in sportspersons. 144.

[16] Vysochin Yu, V., Denisenko Yu, P. and Yatsenko, L.G. (2012) Physiology, medicine, pharmacology, high technologies, the theory, practice. The 4th International Scientifically-Practical Conference, 1, 188-190.

[17] Steg, G. (1964) Efferent muscle innervation and rigidity. Acta Physiologica Scandinavica, 61, 52.

[18] Vallbo, A.B. (1979) Cutaneous mechanoreception. 6th International Congress of Electromyography in Stockholm, Sweden, 14-19.

[19] Vysochin Yu, V. (2001) Myorelaxation in mechanisms of orthopedic injuries. Sport and Nation's Health: Collection of Scientific Works, 74-84. 